\title{
Photodimerization of 1D Ladder Polymer Through Single-Crystal to Single- Crystal Transformation has Effect on Electrical Conductivity
}

Sakhiul Islam, ${ }^{\dagger}$ Joydeep Datta, ${ }^{\ddagger}$ Suvendu Maity, ${ }^{\#}$ Basudeb Dutta, ${ }^{\dagger}$ Samim Khan, ${ }^{\dagger}$ Prasanta Ghosh, Partha Pratim Ray,*‡ and Mohammad Hedayetullah Mir*†

${ }^{\dagger}$ Department of Chemistry, Aliah University, New Town, Kolkata 700 156, India. Email: chmmir@gmail.com

${ }^{\ddagger}$ Department of Physics, Jadavpur University, Jadavpur, Kolkata 700 032, India. Email: partha@phys.jdvu.ac.in

"Department of Chemistry, Jadavpur University, Jadavpur, Kolkata 700 032, India.

${ }^{\Phi}$ Department of Chemistry, R. K. M. Residential College, Narendrapur, Kolkata 700 103, India.

\section{Supporting Information}

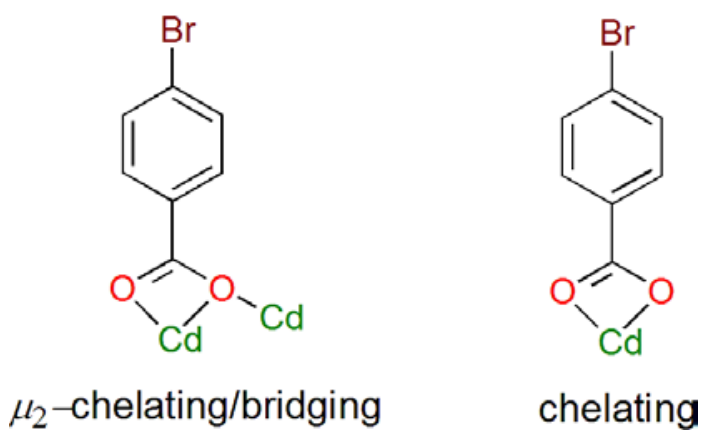

Scheme S1 Coordination modes of $p$-brba in compound $\mathbf{1 .}$ 


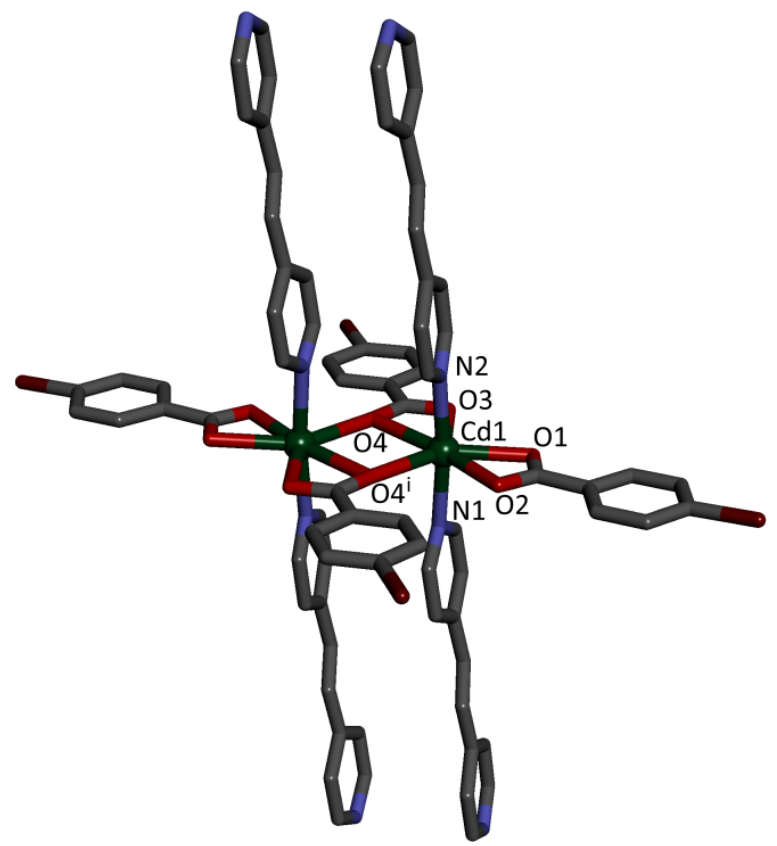

Figure S1. Dimeric repeating unit of $\mathbf{1}$ showing coordination environment of $\mathrm{Cd}(\mathrm{II})$ centre. Symmetry transformation, ${ }^{\mathrm{i}}=-\mathrm{x}, 1-\mathrm{y}, 1-\mathrm{z}$.

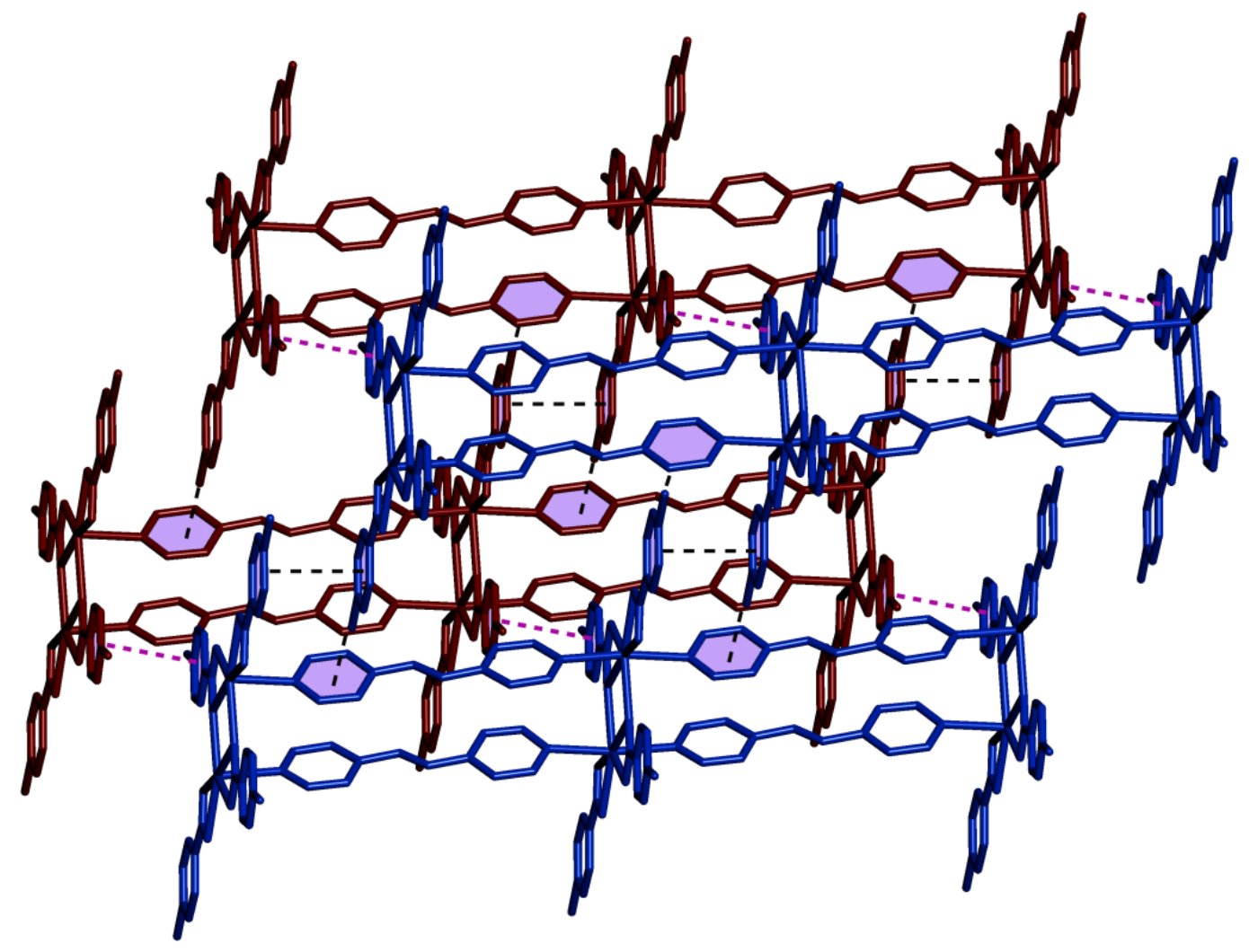

Figure S2. 3D supramolecular architecture of compound $\mathbf{1}$ formed by $\mathrm{Br} \cdots \pi$ and $\pi \cdots \pi$ interactions. 


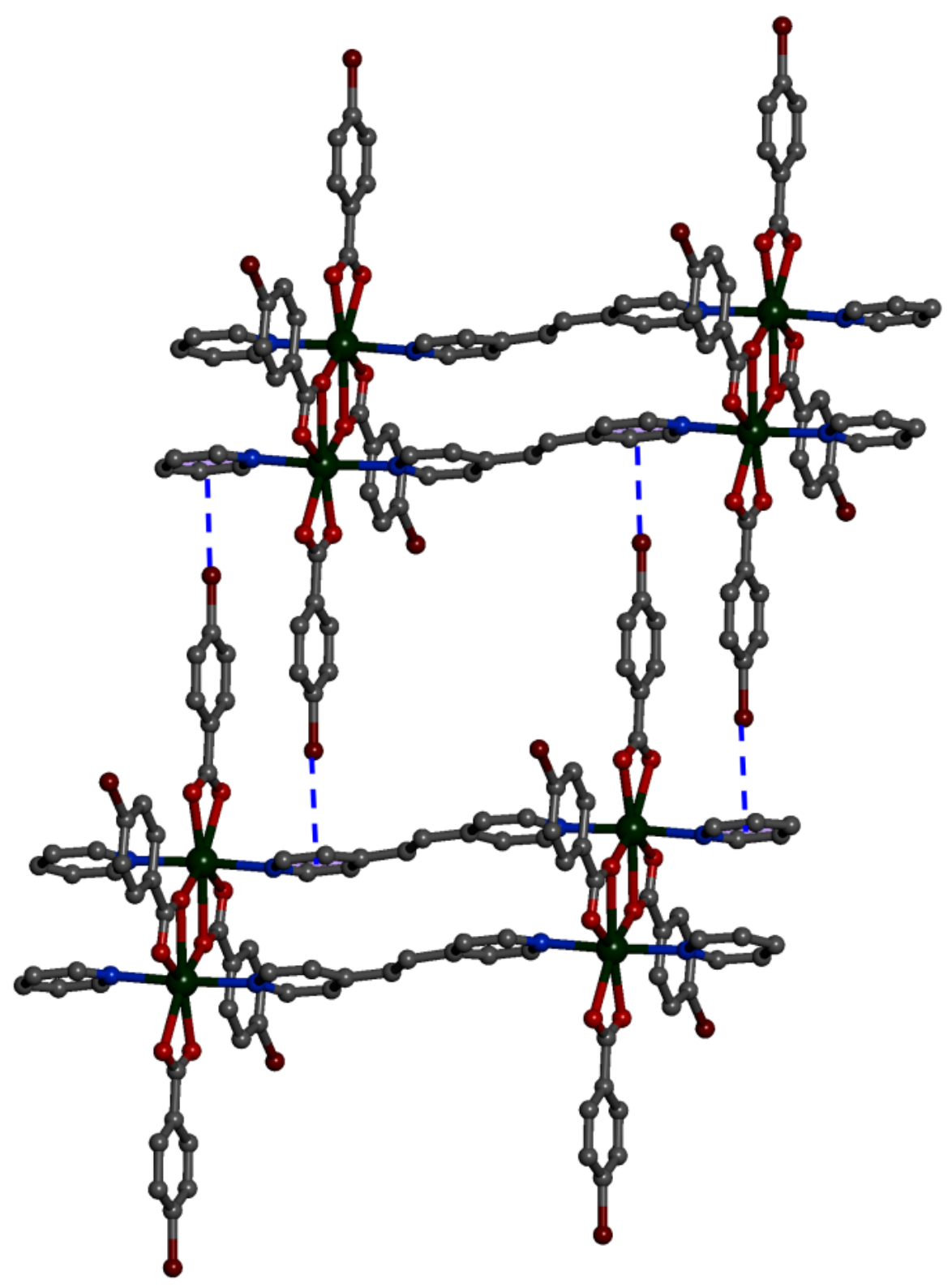

Figure S3. 1D ladder polymer of 1 undergone $\mathrm{Br}^{\cdots} \pi$ interactions. 


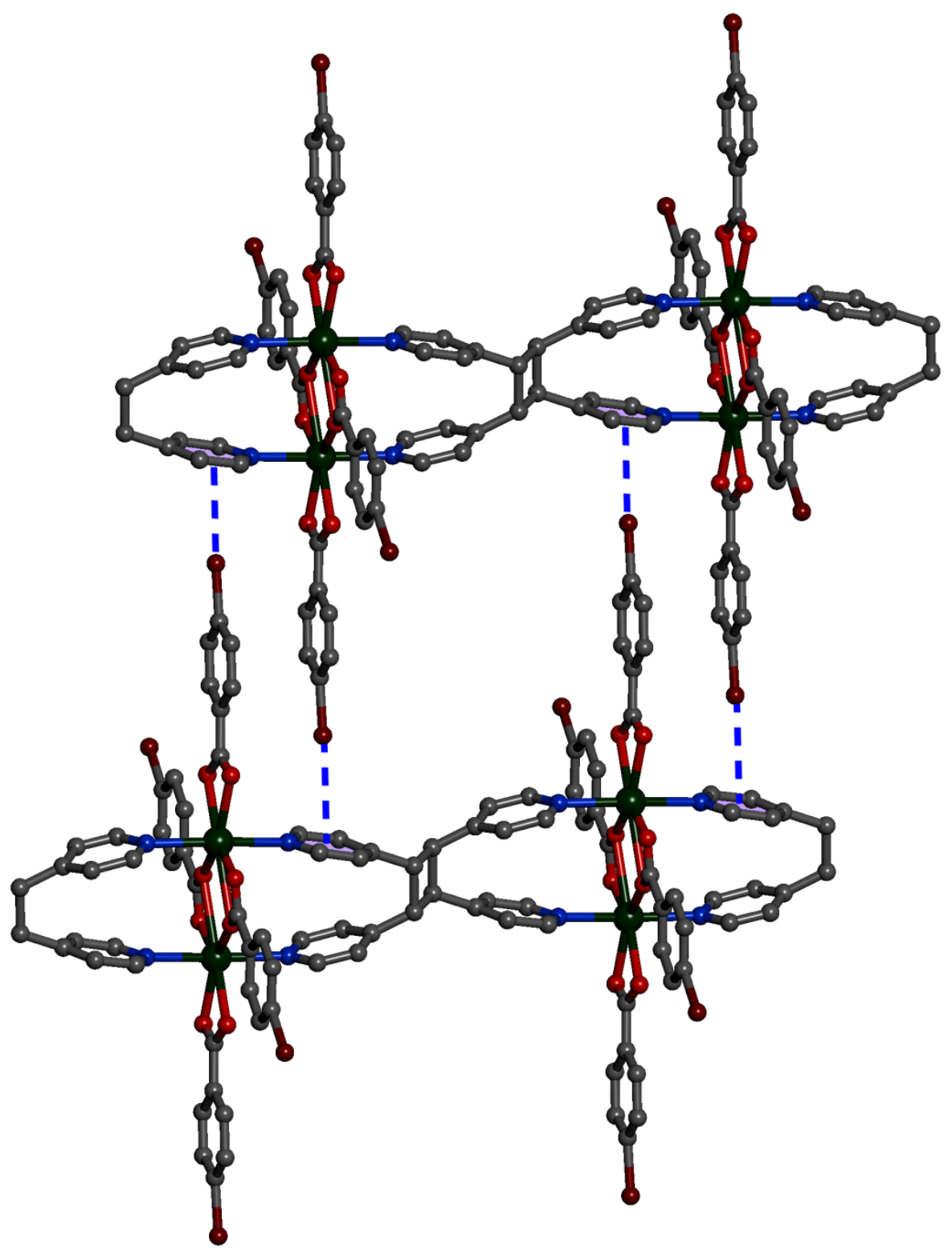

Figure S4. 1D ladder polymer of dinerized product 2 undergone $\mathrm{Br} \cdots \pi$ interactions. 
Table S1 Crystal data and refinement parameters of compound $\mathbf{1}$ and 2.

\begin{tabular}{|c|c|c|}
\hline Compound & 1 & 2 \\
\hline Formula & $\begin{array}{l}\mathrm{C}_{26} \mathrm{H}_{18} \mathrm{Br}_{2} \mathrm{CdN}_{2} \mathrm{O} \\
4\end{array}$ & $\begin{array}{l}\mathrm{C}_{26} \mathrm{H}_{18} \mathrm{Br}_{2} \mathrm{CdN}_{2} \mathrm{O} \\
4\end{array}$ \\
\hline fw & 694.63 & 694.63 \\
\hline cryst syst & triclinic & triclinic \\
\hline space group & $P \overline{1}$ & $P \overline{1}$ \\
\hline$a(\AA)$ & $10.1010(4)$ & $10.3242(15)$ \\
\hline$b(\AA)$ & $11.1154(4)$ & $10.6346(18)$ \\
\hline$c(\AA)$ & $11.7417(5)$ & $12.054(2)$ \\
\hline$\alpha(\operatorname{deg})$ & $88.064(2)$ & $88.205(11)$ \\
\hline$\beta(\operatorname{deg})$ & $71.384(2)$ & $70.829(9)$ \\
\hline$\gamma(\mathrm{deg})$ & $82.305(2)$ & $82.985(11)$ \\
\hline$V\left(\AA^{3}\right)$ & 1238.04(9) & $1240.6(4)$ \\
\hline$Z$ & 2 & 2 \\
\hline$D_{\text {calcd }}\left(\mathrm{g} / \mathrm{cm}^{3}\right)$ & 1.863 & 1.859 \\
\hline$\mu\left(\mathrm{mm}^{-1}\right)$ & 4.145 & 4.136 \\
\hline$\lambda(\AA)$ & 0.71073 & 0.71073 \\
\hline GOF on $F^{2}$ & 1.064 & 1.052 \\
\hline $\operatorname{data}[I>2 \sigma(I)] /$ params & $6083 / 316$ & $4212 / 316$ \\
\hline $\begin{array}{l}\text { final } R \text { indices } \\
{[I>2 \sigma(I)]^{a, b}}\end{array}$ & $\begin{array}{l}R 1=0.0430 \\
w R 2=0.1453\end{array}$ & $\begin{array}{l}R 1=0.0828 \\
w R 2=0.2363\end{array}$ \\
\hline
\end{tabular}


Table S2 Selected bond lengths ( $\AA$ ) in $\mathbf{1}$ and $\mathbf{2}$.

\begin{tabular}{|l|l|l|l|}
\hline \multicolumn{1}{|c|}{$\mathbf{1}$} & \multicolumn{1}{|c|}{$(\AA)$} & \multicolumn{1}{c|}{$\mathbf{2}$} & \multicolumn{1}{c|}{$(\AA)$} \\
\hline $\mathrm{Cd}(1)-\mathrm{O}(1)$ & $2.398(3)$ & $\mathrm{Cd}(1)-\mathrm{O}(1)$ & $2.422(8)$ \\
\hline $\mathrm{Cd}(1)-\mathrm{O}(2)$ & $2.379(3)$ & $\mathrm{Cd}(1)-\mathrm{O}(2)$ & $2.376(9)$ \\
\hline $\mathrm{Cd}(1)-\mathrm{O}(3)$ & $2.335(3)$ & $\mathrm{Cd}(1)-\mathrm{O}(3)$ & $2.380(10)$ \\
\hline $\mathrm{Cd}(1)-\mathrm{O}(4)$ & $2.561(3)$ & $\mathrm{Cd}(1)-\mathrm{N}(1)$ & $2.354(9)$ \\
\hline $\mathrm{Cd}(1)-\mathrm{N}(1)$ & $2.329(3)$ & $\mathrm{Cd}(1)-\mathrm{O}(3) \mathrm{c}$ & $2.691(9)$ \\
\hline $\mathrm{Cd}(1)-\mathrm{N}(2) \mathrm{a}$ & $2.334(3)$ & $\mathrm{Cd}(1)-\mathrm{O}(4) \mathrm{c}$ & $2.292(8)$ \\
\hline $\mathrm{Cd}(1)-\mathrm{O}(4) \mathrm{b}$ & $2.359(3)$ & $\mathrm{Cd}(1)-\mathrm{N}(2) \mathrm{c}$ & $2.336(9)$ \\
\hline
\end{tabular}

Symmetry transformations used to generate equivalent atoms: $\mathrm{a}=1+\mathrm{x},-1+\mathrm{y}, \mathrm{z} ; \mathrm{b}=2-\mathrm{x}, 1-\mathrm{y}, 1-\mathrm{z}$;

$$
c=1-x, 1-y, 2-z
$$


Table S3 Selected bond bond angles $\left(^{\circ}\right)$ in $\mathbf{1}$.

\begin{tabular}{|l|l|l|l|}
\hline $\mathrm{O}(1)-\mathrm{Cd}(1)-\mathrm{O}(2)$ & $54.53(12)$ & $\mathrm{O}(3)-\mathrm{Cd}(1)-\mathrm{N}(1)$ & $93.54(12)$ \\
\hline $\mathrm{O}(1)-\mathrm{Cd}(1)-\mathrm{O}(3)$ & $85.11(11)$ & $\mathrm{O}(3)-\mathrm{Cd}(1)-\mathrm{C}(26)$ & $112.10(12)$ \\
\hline $\mathrm{O}(1)-\mathrm{Cd}(1)-\mathrm{O}(4)$ & $137.64(11)$ & $\mathrm{O}(3)-\mathrm{Cd}(1)-\mathrm{N}(2) \mathrm{a}$ & $90.96(12)$ \\
\hline $\mathrm{O}(1)-\mathrm{Cd}(1)-\mathrm{N}(1)$ & $96.87(11)$ & $\mathrm{O}(3)-\mathrm{Cd}(1)-\mathrm{O}(4) \mathrm{b}$ & $128.74(11)$ \\
\hline $\mathrm{O}(1)-\mathrm{Cd}(1)-\mathrm{C}(26)$ & $27.07(12)$ & $\mathrm{O}(4)-\mathrm{Cd}(1)-\mathrm{N}(1)$ & $88.76(11)$ \\
\hline $\mathrm{O}(1)-\mathrm{Cd}(1)-\mathrm{N}(2) \mathrm{a}$ & $88.98(11)$ & $\mathrm{O}(4)-\mathrm{Cd}(1)-\mathrm{C}(26)$ & $164.68(12)$ \\
\hline $\mathrm{O}(1)-\mathrm{Cd}(1)-\mathrm{O}(4) \mathrm{b}$ & $145.83(11)$ & $\mathrm{O}(4)-\mathrm{Cd}(1)-\mathrm{N}(2) \mathrm{a}$ & $89.62(11)$ \\
\hline $\mathrm{O}(2)-\mathrm{Cd}(1)-\mathrm{O}(3)$ & $139.45(12)$ & $\mathrm{O}(3)-\mathrm{Cd}(1)-\mathrm{O}(4) \mathrm{b}$ & $76.19(11)$ \\
\hline $\mathrm{O}(2)-\mathrm{Cd}(1)-\mathrm{O}(4)$ & $167.79(12)$ & $\mathrm{N}(1)-\mathrm{Cd}(1)-\mathrm{C}(26)$ & $92.79(11)$ \\
\hline $\mathrm{O}(2)-\mathrm{Cd}(1)-\mathrm{N}(1)$ & $88.27(12)$ & $\mathrm{N}(1)-\mathrm{Cd}(1)-\mathrm{N}(2) \mathrm{a}$ & $172.91(11)$ \\
\hline $\mathrm{O}(2)-\mathrm{Cd}(1)-\mathrm{C}(26)$ & $27.46(13)$ & $\mathrm{O}(4) \mathrm{b}-\mathrm{Cd}(01)-\mathrm{N}(1)$ & $86.69(11)$ \\
\hline $\mathrm{O}(2)-\mathrm{Cd}(1)-\mathrm{N}(2) \mathrm{a}$ & $91.88(12)$ & $\mathrm{N}(2) \mathrm{a}-\mathrm{Cd}(1)-\mathrm{C}(26)$ & $90.60(11)$ \\
\hline $\mathrm{O}(2)-\mathrm{Cd}(1)-\mathrm{O}(4) \mathrm{b}$ & $91.81(12)$ & $\mathrm{O}(4) \mathrm{b}-\mathrm{Cd}(1)-\mathrm{C}(26)$ & $119.10(12)$ \\
\hline $\mathrm{O}(3)-\mathrm{Cd}(1)-\mathrm{O}(4)$ & $52.59(11)$ & $\mathrm{O}(4) \mathrm{b}-\mathrm{Cd}(1)-\mathrm{N}(2) \mathrm{a}$ & $86.21(11)$ \\
\hline
\end{tabular}

Symmetry transformations used to generate equivalent atoms: $\mathrm{a}=1+\mathrm{x},-1+\mathrm{y}, \mathrm{z} ; \mathrm{b}=2-\mathrm{x}, 1-\mathrm{y}, 1-\mathrm{z}$ 
Table S4 Selected bond angles $\left(^{\circ}\right)$ in 2.

\begin{tabular}{|l|l|l|l|}
\hline $\mathrm{O}(1)-\mathrm{Cd}(1)-\mathrm{O}(2)$ & $54.2(3)$ & $\mathrm{O}(3)-\mathrm{Cd}(1)-\mathrm{C}(8)$ & $118.3(3)$ \\
\hline $\mathrm{O}(1)-\mathrm{Cd}(1)-\mathrm{O}(3)$ & $144.8(3)$ & $\mathrm{O}(3)-\mathrm{Cd}(1)-\mathrm{O}(3) \mathrm{c}$ & $72.1(3)$ \\
\hline $\mathrm{O}(1)-\mathrm{Cd}(1)-\mathrm{N}(1)$ & $94.8(3)$ & $\mathrm{O}(3)-\mathrm{Cd}(1)-\mathrm{O}(4) \mathrm{c}$ & $123.1(3)$ \\
\hline $\mathrm{O}(1)-\mathrm{Cd}(1)-\mathrm{C}(8)$ & $26.8(3)$ & $\mathrm{O}(3)-\mathrm{Cd}(1)-\mathrm{N}(2) \mathrm{c}$ & $86.7(3)$ \\
\hline $\mathrm{O}(1)-\mathrm{Cd}(1)-\mathrm{O}(3) \mathrm{c}$ & $141.7(3)$ & $\mathrm{N}(1)-\mathrm{Cd}(1)-\mathrm{C}(8)$ & $92.1(3)$ \\
\hline $\mathrm{O}(1)-\mathrm{Cd}(1)-\mathrm{O}(4) \mathrm{c}$ & $91.8(3)$ & $\mathrm{O}(3) \mathrm{c}-\mathrm{Cd}(1)-\mathrm{N}(1)$ & $96.0(3)$ \\
\hline $\mathrm{O}(1)-\mathrm{Cd}(1)-\mathrm{N}(2) \mathrm{c}$ & $88.5(3)$ & $\mathrm{O}(4) \mathrm{c}-\mathrm{Cd}(1)-\mathrm{N}(1)$ & $92.1(3)$ \\
\hline $\mathrm{O}(2)-\mathrm{Cd}(1)-\mathrm{O}(3)$ & $91.8(3)$ & $\mathrm{N}(1)-\mathrm{Cd}(1)-\mathrm{N}(2) \mathrm{c}$ & $175.7(3)$ \\
\hline $\mathrm{O}(2)-\mathrm{Cd}(1)-\mathrm{N}(1)$ & $88.5(3)$ & $\mathrm{O}(3) \mathrm{c}-\mathrm{Cd}(1)-\mathrm{C}(8)$ & $167.0(3)$ \\
\hline $\mathrm{O}(2)-\mathrm{Cd}(1)-\mathrm{C}(8)$ & $27.4(3)$ & $\mathrm{O}(4) \mathrm{c}-\mathrm{Cd}(1)-\mathrm{C}(8)$ & $118.6(3)$ \\
\hline $\mathrm{O}(2)-\mathrm{Cd}(1)-\mathrm{O}(3) \mathrm{c}$ & $162.7(3)$ & $\mathrm{N}(2) \mathrm{b}-\mathrm{Cd}(1)-\mathrm{C}(8)$ & $89.5(3)$ \\
\hline $\mathrm{O}(2)-\mathrm{Cd}(1)-\mathrm{O}(4) \mathrm{c}$ & $145.8(3)$ & $\mathrm{O}(3) \mathrm{c}-\mathrm{Cd}(1)-\mathrm{O}(4) \mathrm{c}$ & $51.1(3)$ \\
\hline $\mathrm{O}(2)-\mathrm{Cd}(1)-\mathrm{N}(2) \mathrm{c}$ & $91.7(3)$ & $\mathrm{O}(3) \mathrm{c}-\mathrm{Cd}(1)-\mathrm{N}(2) \mathrm{c}$ & $83.2(3)$ \\
\hline $\mathrm{O}(3)-\mathrm{Cd}(1)-\mathrm{N}(1)$ & $89.0(3)$ & $\mathrm{O}(4) \mathrm{c}-\mathrm{Cd}(1)-\mathrm{N}(2) \mathrm{c}$ & $90.6(3)$ \\
\hline
\end{tabular}

Symmetry transformations used to generate equivalent atoms: c = 1-x, 1-y, 2-z 


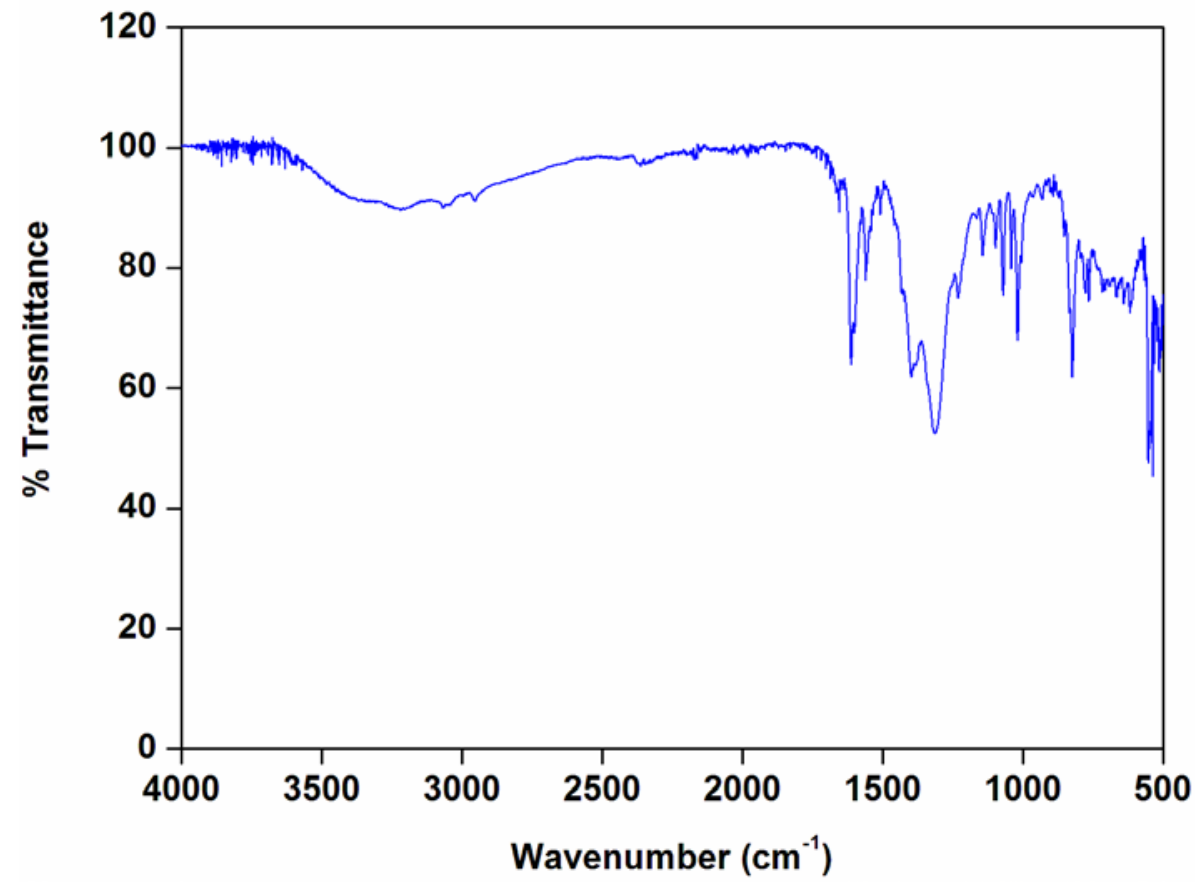

Figure S5. FT-IR spectrum of compound 1.

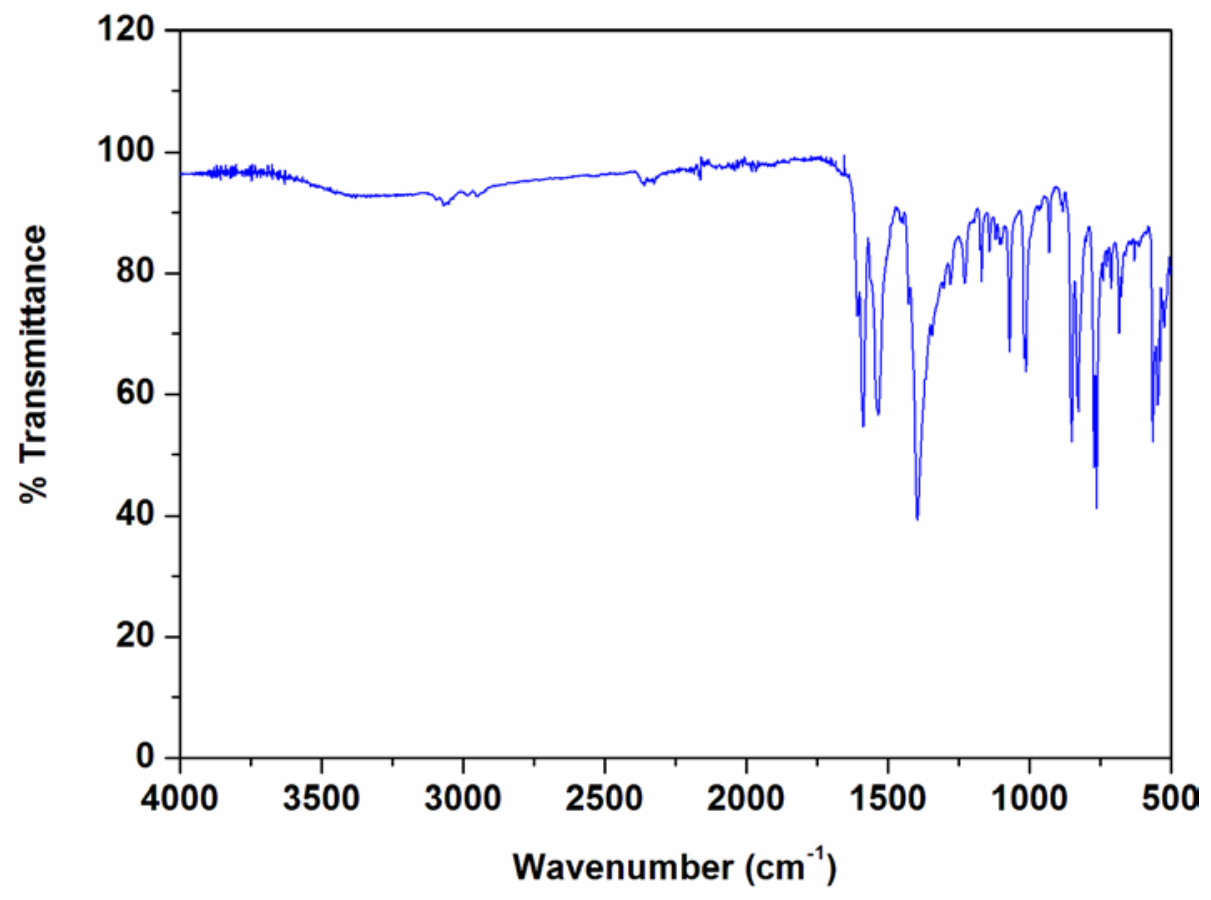

Figure S6. FT-IR spectrum of compound 2. 


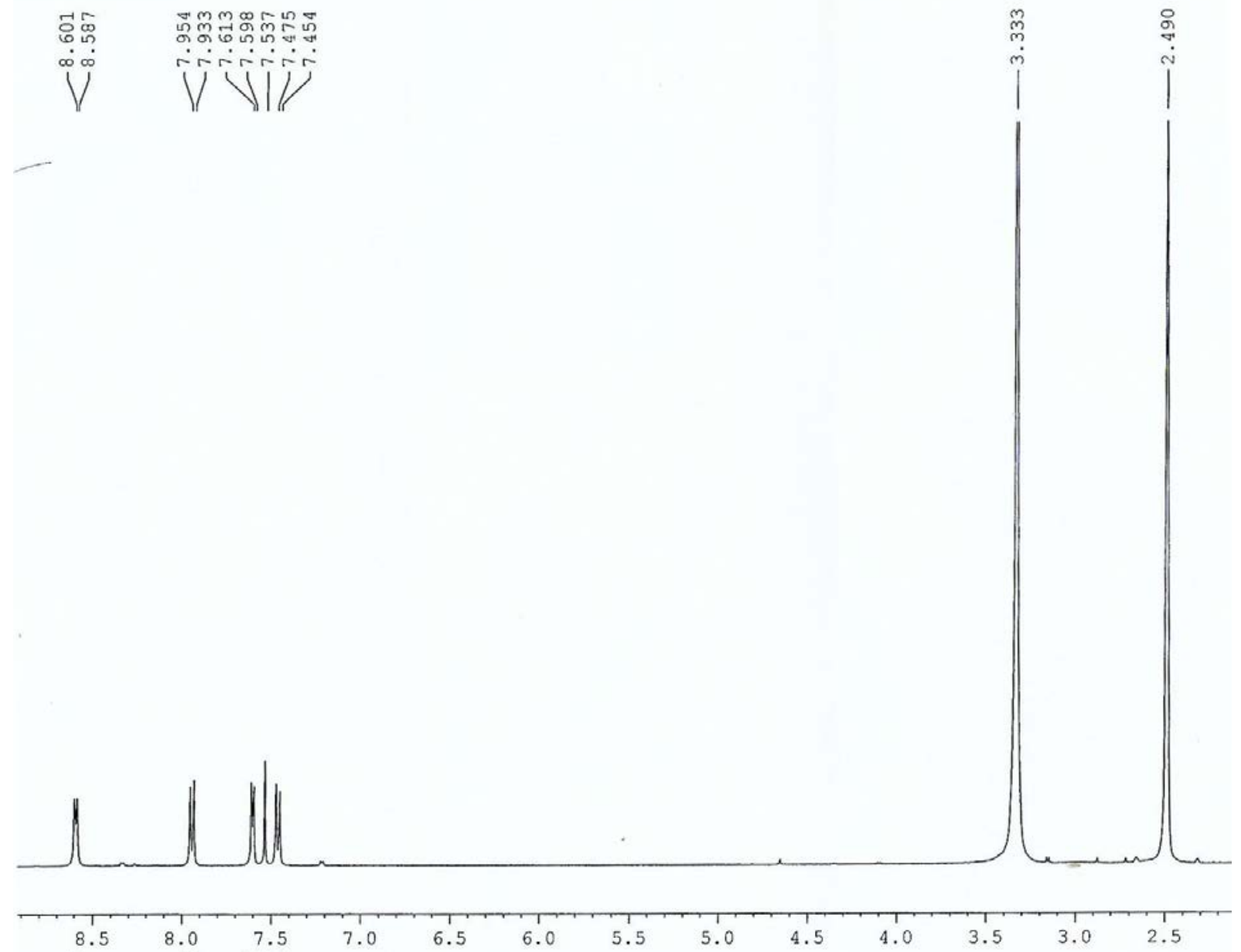

Figure S7. ${ }^{1} \mathrm{H}$ NMR spectrum of 1 in $d_{6}$-DMSO. 


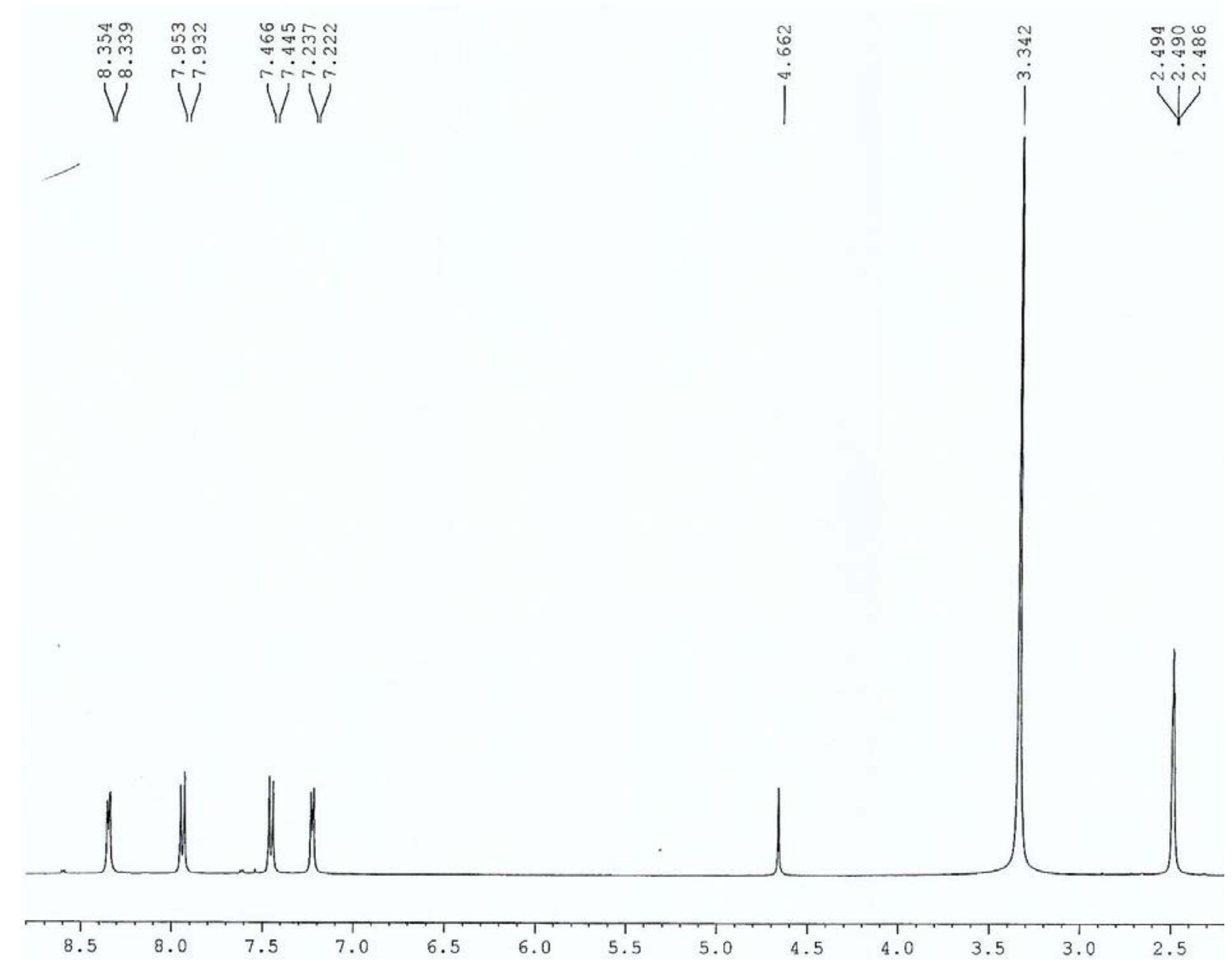

Figure S8. ${ }^{1} \mathrm{H}$ NMR spectrum of 2 in $d_{6}-\mathrm{DMSO}$.

\section{Frequency Dependent Capacitance Measurements}

Figure S9 illustrates the frequency dependent capacitance of the synthesized compounds. We have observed that the capacitance of the materials is high at low frequency and saturated at high frequency. We have used this saturated capacitance value and derived the relative permittivity using the following the relation

$$
\varepsilon_{\mathrm{r}}=\frac{\mathrm{C}_{\mathrm{sat}} \mathrm{L}}{\varepsilon_{0} \mathrm{~A}}
$$


Here, $C_{\text {sat }}$ is the saturated capacitance, $L$ is the film thickness $(\sim 10 \mu \mathrm{m})$ and $A$ is the effective contact area $\left(7.056 \times 10^{-6} \mathrm{~m}^{2}\right)$ of the pellet of synthesized composite. The values of $\varepsilon_{r}$ are measured as 2.4 and 1.2 for compound $\mathbf{1}$ and $\mathbf{2}$, respectively.

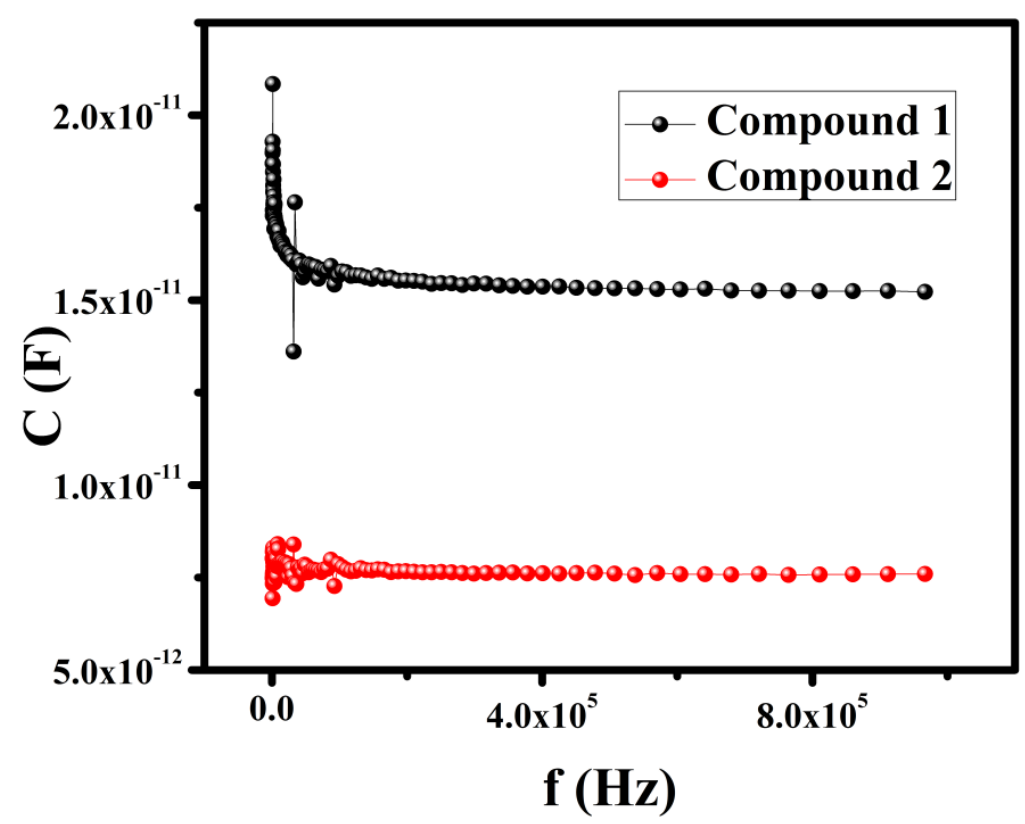

Figure S9. Capacitance vs. frequency plot for the compound 1 and 2. 